\title{
Light-induced phase transitions of colloidal monolayers with crystalline order
}

\author{
Lamiss Zaidouny, ${ }^{a}$ Thomas Bohlein, ${ }^{a}$ Roland Roth ${ }^{\mathrm{b}}$ and Clemens Bechinger ${ }^{\star a c}$ \\ We experimentally study the phase behavior of a charge-stabilized two-dimensional colloidal crystal which \\ is subjected to a one-dimensional periodic light field. Such light fields are created by a scanned optical line \\ tweezer which allows the variation of the periodicity without optical realignments. In order to realize a \\ wide range of line spacings relative to the lattice constant, we use a suspension of silica particles in \\ bromobenzene. This colloidal system has a Debye screening length of about $4.6 \mu \mathrm{m}$ which results in the \\ formation of crystals with lattice constants up to $20 \mu \mathrm{m}$. Because the refractive index of bromobenzene \\ is larger than that of the colloids, optical gradient forces lead to the attraction of particles at regions \\ where the intensity is smallest. Depending on the depth and periodicity of the optical potential, we \\ observe the light-induced assembly of colloids into triangular, rhombic and square phases.
}

\section{Introduction}

The investigation of the phase behavior in colloidal monolayers has a long history in providing deep insights into how matter organizes to form liquid or crystalline states. ${ }^{1,2}$ In particular, phase transitions in two-dimensional systems have attracted considerable attention, because such systems are also of great technological importance. ${ }^{3,4}$ Often, the transition between a liquid (disordered) and a crystalline (ordered) state is induced by variations in the particle pair potential. ${ }^{5,6}$ In addition, structural phase transitions can be also triggered by external periodic fields which enhance the long-range positional order. While the latter is obvious for two-dimensional substrate potentials which localize the particles at well-defined positions in the sample plane, ${ }^{7-9}$ it is less obvious that a one-dimensional, periodic substrate potential can also induce two-dimensional order in a colloidal system. The first experiments in this direction have been carried out by Chowdhury, Ackerson and Clark, who demonstrated the freezing of a colloidal liquid into a hexagonal crystalline state by application of an optical interference pattern of periodically spaced lines. When they tuned the distance of the laser lines to $\boldsymbol{d}=\boldsymbol{a} \sqrt{3} / 2$ with $\boldsymbol{a}$ the mean particle distance, they observed a phase transition from a liquid into a hexagonal phase as the laser intensity was increased (laser-induced freezing). ${ }^{\mathbf{1 0}}$ Later, it has been demonstrated that a further increase of the laser intensity can lead to reentrant behavior from a hexagonal phase to a liquid. This is found to be in agreement with theoretical and numerical studies. ${ }^{11,12}$

a2. Physikalisches Institut, Universität Stuttgart, D-70550 Stuttgart, Germany. E-mail: c.bechinger@physik.uni-stuttgart.de

${ }^{b}$ Institut für Theoretische Physik, Universität Tübingen, D-72076 Tübingen, Germany ${ }^{c}$ MPI für Intelligente Systeme, D-70569 Stuttgart, Germany
In contrast to the above experiments where the initial phase (i.e. the phase which formed in the absence of a light pattern) was a colloidal liquid, here we investigate the response of a charge-stabilized colloidal crystal with lattice constant $\boldsymbol{a}$ to a one-dimensional light field with periodicity $\boldsymbol{d}$. To obtain a large range of $\boldsymbol{d} / \boldsymbol{a}$ values, we have used a colloidal suspension of silica particles in bromobenzene which leads to lattice constants up to $20 \mu \mathrm{m}$. Depending on the intensity, periodicity and even the width of the light potential, we observe a variety of different colloidal phases with triangular, rhombic and square order.

\section{Materials and methods}

\subsection{Colloidal suspension}

In our experiments we use charged silica spheres with diameter $\sigma=3.72 \mu \mathrm{m}$ and a polydispersity less than $5 \%$ suspended in an organic solvent, namely bromobenzene $\left(\mathrm{C}_{6} \mathrm{H}_{5} \mathrm{Br}\right)$. These colloids were purchased as an aqueous suspension from Microparticles $\mathrm{GmbH}$ with a standard deviation size of $\mathrm{SD}=$ $0.08 \mu \mathrm{m}$ and mass concentration of $5 \% \mathrm{w} / \mathrm{v}$. These silica spheres are hydrophilic and when placed in contact with water acquire a negative charge due to the dissociation of the silanol group $\mathrm{SiOH}$ to $\mathrm{SiO}^{-}$(surface charge) and $\mathrm{H}^{+}$as counter ions. ${ }^{13}$ Before the colloids are transferred to bromobenzene, the following steps have to be done carefully to ensure monodispersity and stability of the colloids. First, a $100 \mu \mathrm{l}$ sample is prepared in a glass micro tube where the original solvent is replaced by highly deionized water with an ionic conductivity of $0.06 \mu \mathrm{S} \mathrm{cm}^{-1}$. This process helps to decrease the number of ions in the solvent, thus enhancing the electrostatic stability of the suspension. Next, the suspension is centrifuged for 2 minutes at $2000 \mathrm{rpm}$ to separate the water from the particles. The water is then taken out and replaced by deionized water. This step is repeated 
several times before the suspension is sonicated for few minutes. Sonication helps to release coagulated particles which occurred due to the centrifugation process and therefore insures monodispersity in the suspension. Then, the water is evaporated from the suspension under vacuum conditions at room temperatures. After this process, a thin adsorbed layer is left on the surface of silica particles. ${ }^{13,14}$ We add $1 \mathrm{ml}$ of bromobenzene to the dried sample and place it in an electrical shaker for several hours to separate particles that aggregated during the drying process. At this stage sonication is avoided since this may cause the degradation of bromobenzene into its constituents, i.e. benzene and bromine. ${ }^{15}$ After the bromobenzene suspension is left for half an hour, aggregates sediment towards the bottom where they can be easily removed. Finally, around $100 \mu \mathrm{l}$ of the aggregate-free suspension is injected into a sample cell (Hellma) which consists of a fused silica cuvette with $200 \mu \mathrm{m}$ height. The cell is then sealed tightly to avoid contact with air since it will significantly increase the ionic concentration in the solvent as a function of time. In a closed cell, the suspension maintains its properties for a period of two months.

Bromobenzene is an organic solvent with a refractive index $n$ $=1.56$ and a polarity close to the limit of that of apolar liquids. ${ }^{16}$ Since the density of silica particles is larger than that of bromobenzene, then particles sediment toward the bottom surface of the cell. Therefore, for particle densities in the range of $\Phi=0.004-0.006 \mu \mathrm{m}^{-2}$ the colloidal system form a two-dimensional charge-stabilized crystal where due to the large Debye screening length (see below) the triangular crystal have lattice constants up to $20 \mu \mathrm{m}$. Because the out of phase fluctuations account to less than $100 \mathrm{~nm}$, even though particles are 3d spheres, they can be considered as effective two dimensional.

In order to determine the pair interaction of our experimental system, we prepared several low density samples $\left(\Phi=0.002 \mu \mathrm{m}^{-2}\right)$ and measured the pair correlation function $g(r)$. A direct inversion of $g(r)$ for two-dimensional systems is not possible, therefore we performed some model density functional theory (DFT) calculations ${ }^{17}$ for a two-dimensional system of disks that interact via a hard-core Yukawa potential of the form:

$$
U(r) / k_{\mathrm{B}} T=A / r \exp (-\kappa r), \text { for } r>\sigma
$$

with $U(r)=\infty$, for $r<\sigma, A=\left(Z^{*}\right)^{2} \lambda_{\mathrm{B}}[\exp (\kappa \sigma / 2) /(1+(\kappa \sigma / 2))]^{2}$. Here, $r$ is the center-to-center particle distance, $Z^{*}$ the effective surface charge, $\lambda_{\mathrm{B}}$ the Bjerrum length, $\kappa$ the inverse screening length and $\sigma$ the diameter of the particles. In the case of bromobenzene (dielectric constant $\varepsilon=5.6$ ) the Bjerrum length is $10 \mathrm{~nm}$.

We employed a recently developed $\mathrm{DFT}^{\mathbf{1 8}}$ for the hard disk reference system and a perturbation theory treatment for the soft Yukawa repulsion. ${ }^{19}$ The Yukawa parameters were obtained by combining two observations: from the experimental particle trajectories we obtained the average number density and its fluctuation in a sub-volume of our system. From this, it is possible to determine the compressibility of the fluid, ${ }^{19}$ which for the DFT approach results in an equation that combines the two Yukawa parameters $A$ and $\kappa$. Comparing the measured to the computed $g(r)$, the latter determined from test particle route DFT calculations, we obtained the Yukawa parameters, under the constraint of the above obtained compressibility. Best agreement between experiment and theory was found for $A=186 \mu \mathrm{m}$ and $\kappa^{-1}=4.6 \mu \mathrm{m}$ which leads to an effective surface charge $Z^{*}=128$. This small value seems to be reasonable in face of the low polarity solvent. Therefore a variation of the particle density within the range mentioned earlier does not considerably change the ionic strength. In addition, the contribution of the colloidal monolayer to the overall counter ion concentration is rather small since the height of our sample cell is $200 \mu \mathrm{m}$.

\subsection{Creation of one-dimensional periodic light potentials}

One dimensional, periodic potentials were formed by scanning the line focus of a laser beam $(\lambda=532 \mathrm{~nm})$ across the sample. As a result of optical forces ${ }^{20-23}$ such light patterns act as onedimensional potential landscapes acting on the particles. Experimentally, such light fields were created by deflection of a laser beam by a galvanostatically driven mirror and imaging its pivot point by a telescope to a cylindrical lens. The latter forms the initial spherical cross-section of the laser beam (about $3 \mathrm{~mm}$ diameter) into a line with half width of $3.25 \mu \mathrm{m}$ which is then imaged into the sample plane (Fig. 1a and b). In order to create arbitrary line patterns with generally non-sinusoidal shapes, we have scanned the line focus in jerky leaps across the sample plane. This is achieved by application of a step like voltage protocol to the scanner as schematically shown in Fig. 1d. Such protocols are characterized by the time interval $\Delta t$, which corresponds to the resting time of the laser beam being set to about $400 \mu \mathrm{s}$ in our experiments. The height of the steps $\Delta V$ determines the spacing between adjacent laser lines which was varied between approximately $6-12 \mu \mathrm{m}$. As a result, the laser line starts from $x=0$, goes to one end $(+x)$ in one direction then follows back the same path in the opposite direction $(-x)$ passing by $x=0$ again. By this it forms a complete cycle by continuously jumping with the same distance $\boldsymbol{d}$. The repetition time of each cycle was set to $T=0.025 \mathrm{~s}(40 \mathrm{~Hz})$. During that time the colloidal particle diffuses over a distance $L$ determined from the mean square displacement $L^{2}=\left\langle\Delta x^{2}\right\rangle=2 D T$, with $D=$ $k_{\mathrm{B}} T / 6 \pi \eta R$ the diffusion coefficient (with $R$ the radius of the particle, $\eta$ the viscosity of the solvent, $T$ the temperature, and $k_{\mathrm{B}}$ the Boltzmann constant). Under our experimental conditions, we obtain $D=0.104 \mu \mathrm{m}^{2} \mathrm{~s}^{-1}$ yielding $L=0.072 \mu \mathrm{m}$ which is less than $2 \%$ of the half width of the optical trap. Accordingly, our light potentials can be regarded as quasi-static. The accuracy of the line position is determined to about $0.03 \mu \mathrm{m}$. Compared to light potentials created by interfering laser beams ${ }^{\mathbf{1 0 , 1 2}}$ this method allows the in situ control of the periodicity without any optical realignment. In addition this method allows the creation of periodic and non-periodic light patterns. This is exemplarily shown in Fig. 1c where we created a quasiperiodic Dodecanacci sequence ${ }^{24}$ being closely related to 12 -fold quasicrystals. This sequence is comprised of two length scales, $L$ and $S$ with $L / S=(1+\sqrt{3}) / 2$. 
(a)

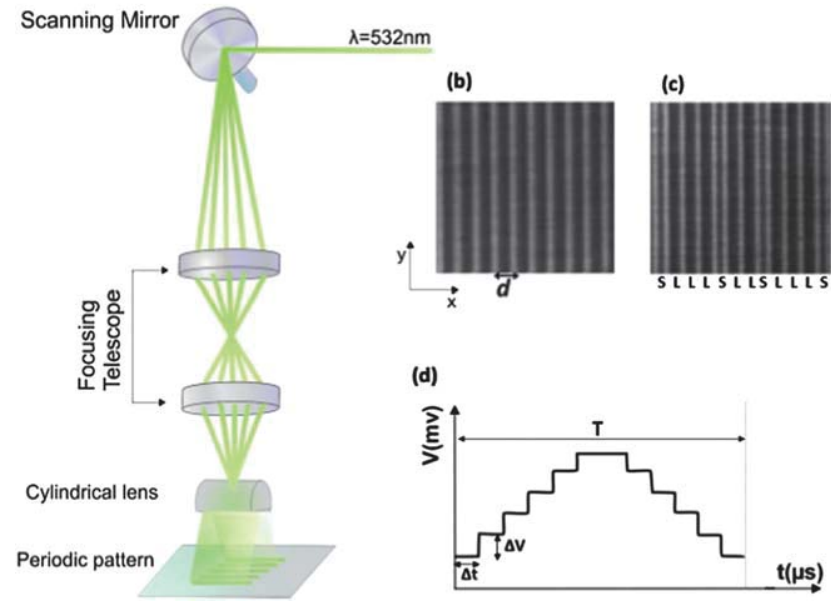

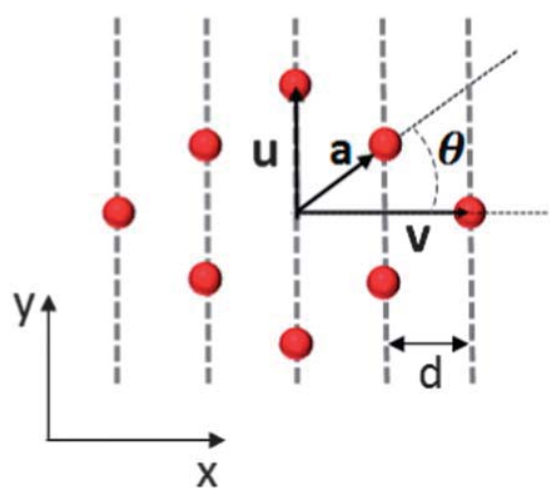

Fig. 3 Center region of the $g(x, y)$ illustrating the average nearest particle distance $v$ and $u$ along the $x$ and $y$ axes respectively, with an average particle distance $\boldsymbol{a}$ along symmetry lines making angle $\theta= \pm \tan ^{-1}(\xi)$ with the $x$-axis.

Fig. 1 (a) Schematic sketch of the experimental setup with a laser beam which is first reflected on a galvanostatically driven mirror and then directed through a telescope to a cylindrical lens. (b) Snapshot of the periodic light pattern taken at the sample position characterized by a period $\boldsymbol{d}$. (c) Snapshot of a quasiperiodic pattern characterized by two length scales $L$ and $S$. (d) Typical step-like voltage input supplied to the mirror which leads to the intensity pattern shown in (b).

Since the refractive index of bromobenzene is larger than that of silica $(n=1.42)$, the colloidal particles will be expelled from the high intensity regions and attracted towards locations where the laser pattern has its smallest intensity. To have a quantitative estimate about the particle-light interaction, we measured the particle density distribution of a highly diluted colloidal suspension $\left(\Phi=0.0003 \mu \mathrm{m}^{-2}\right)$ when subjected to the

Table 1 Characterization of different crystalline phases by different order parameters as defined in the text

\begin{tabular}{llll}
\hline Crystal phase $\backslash$ parameter & $\boldsymbol{d} / \boldsymbol{a}$ & $\xi=u / v$ & $\theta=\tan ^{-1}(\xi)$ \\
\hline Triangular I & 0.86 & $0.554,0.600$ & $29^{\circ}, 31^{\circ}$ \\
Rhombic I & $0.85,0.72$ & $0.600,0.965$ & $31^{\circ}, 44^{\circ}$ \\
Square & 0.71 & $0.965,1.036$ & $44^{\circ}, 46^{\circ}$ \\
Rhombic II & $0.69,0.51$ & $1.036,1.000$ & $46^{\circ}, 59^{\circ}$ \\
Triangular II & 0.5 & $1.660,1.804$ & $59^{\circ}, 61^{\circ}$
\end{tabular}

light potential. Evaluating the particle probability distribution in the direction perpendicular to the laser lines, and employing Boltzmann statistics we obtain the depth and width of the laser

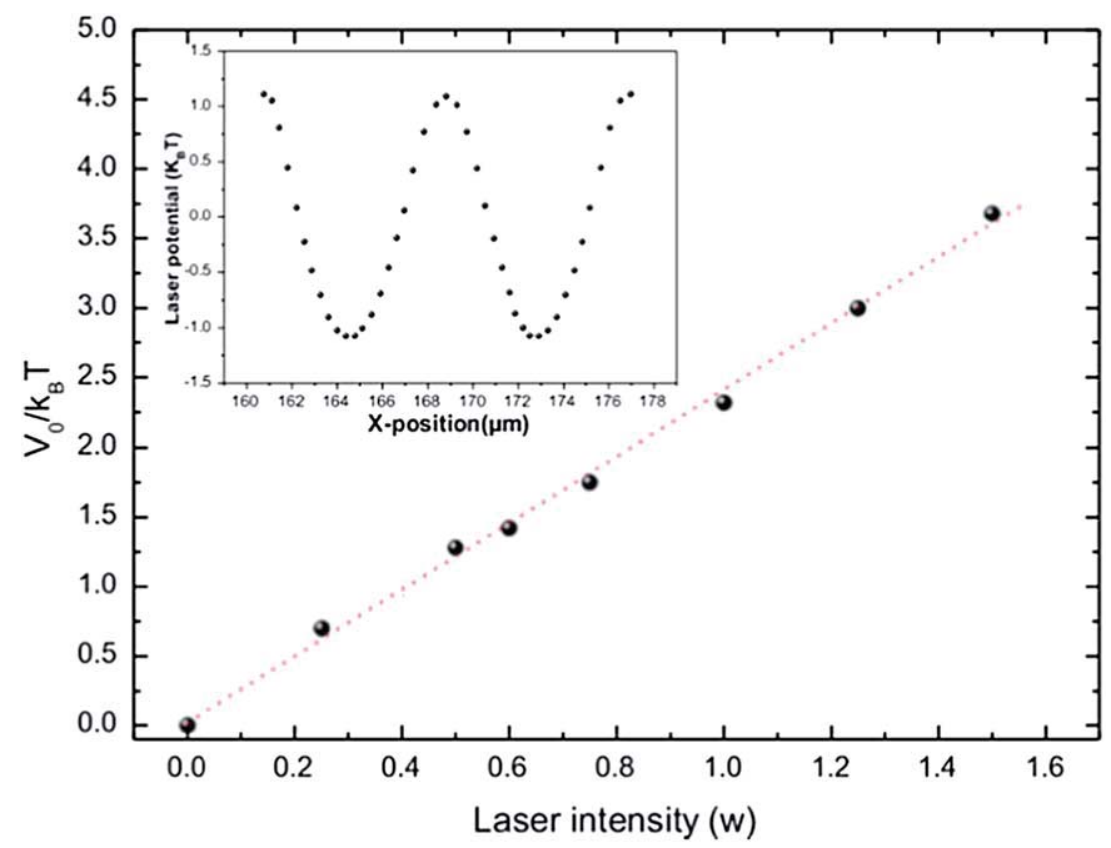

Fig. 2 Amplitude of laser potential acting on colloidal particles of $3.72 \mu \mathrm{m}$ diameter as a function of the incident laser intensity. The symbols correspond to the measured data and the dashed line is a linear fit which indicates the linear relationship between $V_{0}$ and the laser intensity. The inset shows a part of the cross-section of the potential for a line spacing $\boldsymbol{d}=8.2 \mu \mathrm{m}$. It should be emphasized, that in general non-sinusoidal potential cross-sections are obtained. The similarity with a sine function shown here is a result of the Gaussian intensity distribution of the laser line and the specific line spacing chosen. 

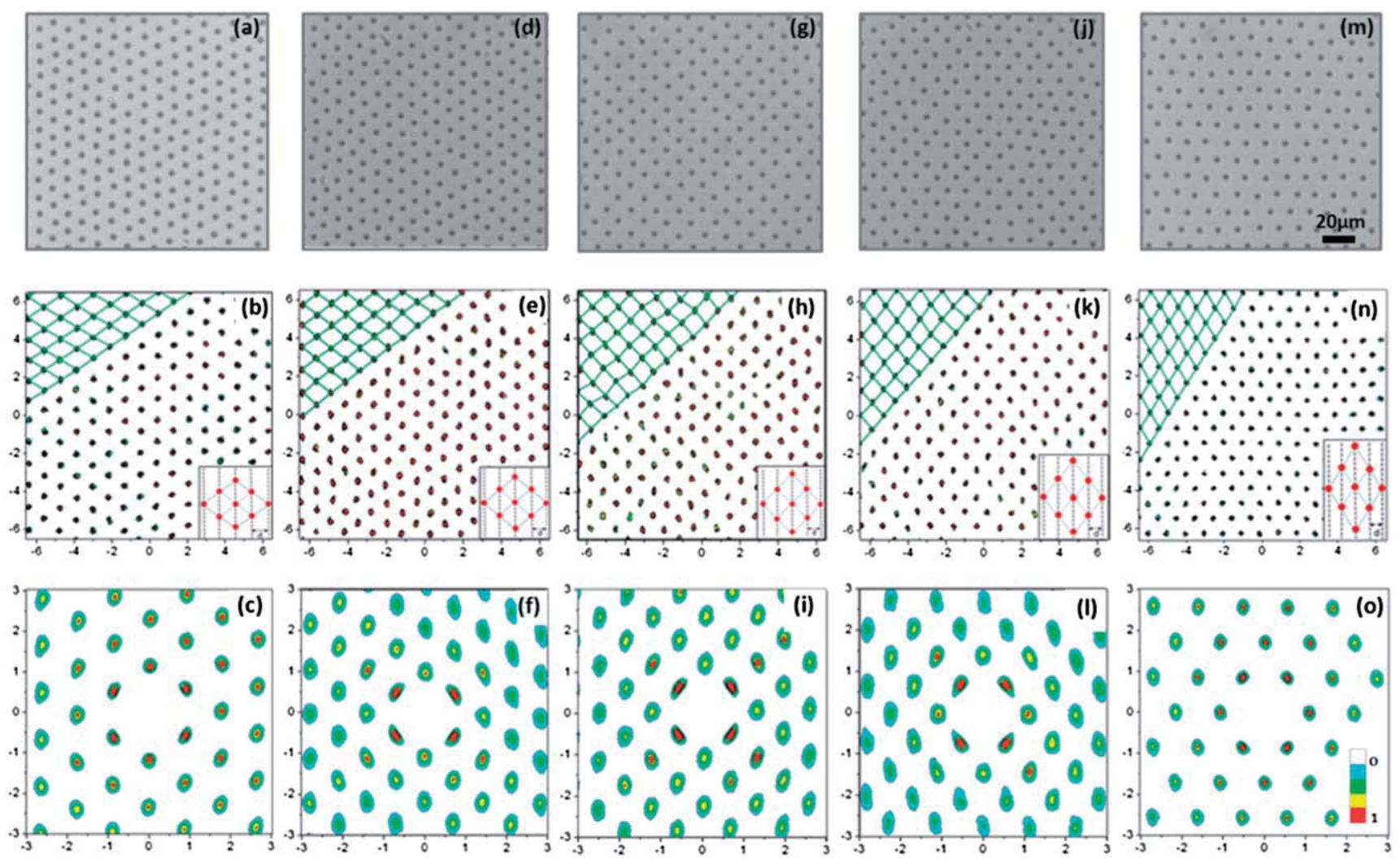

Fig. 4 Snap shots for the different phases are shown at the top while contour plots of the average density distribution function $\rho(x, y)$ are shown at the middle and pair correlation function $g(x, y)$ are shown at the bottom for a potential strength of $V_{0}=7.2 \mathrm{k}_{\mathrm{B}} T$. [a, b and $\mathrm{c}$ ] Triangular I phase at a periodicity $\boldsymbol{d}=11.6 \mu \mathrm{m}$ and $\xi=0.588$, [d, e and f] rhombic I phase at $\boldsymbol{d}=10.4 \mu \mathrm{m}$ and $\xi=0.830$ [g, h and i], square phase at $\boldsymbol{d}=9.58 \mu \mathrm{m}$ and $\xi=0.995$, [j, $\mathrm{k}$ and $\mathrm{l}] \mathrm{rhombic}$ II phase at $\boldsymbol{d}=8.5 \mu \mathrm{m}$ and $\xi=1.217$, $[\mathrm{m}, \mathrm{n}$ and o] triangular II phase at $\boldsymbol{d}=6.75 \mu \mathrm{m}$ and $\xi=1.681$. Green lines in $\rho(x, y)$ help to visualize the structure of each phase. All length scales are normalized by the lattice constant $\mathbf{a}$ of the initial phase.

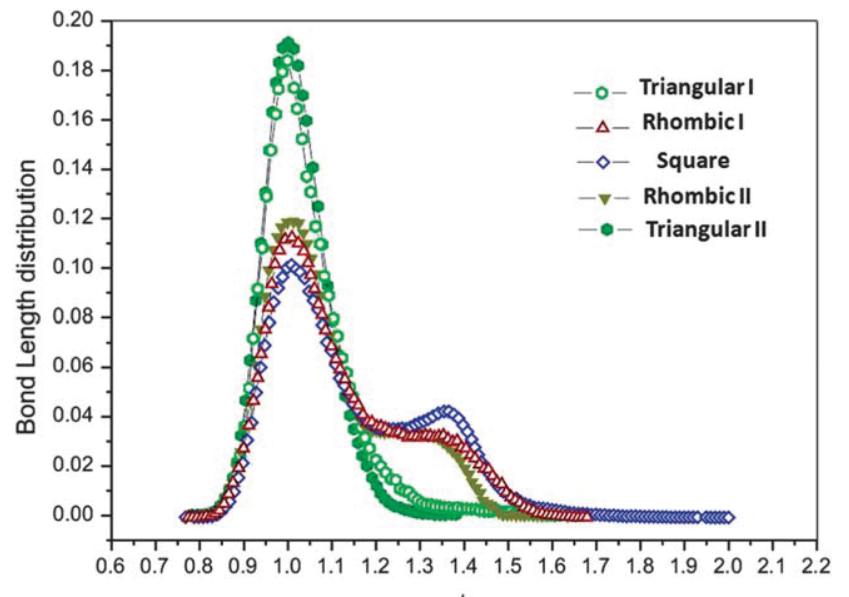

$\mathrm{r} / \mathrm{a}$

Fig. 5 Bond Length distribution for the five phases at $7.2 k_{\mathrm{B}} T$. Triangular I (light green $\square$ ) and triangular II (dark green ) show single peaks while rhombic I (red $\Delta$ ), rhombic II (brown $\boldsymbol{\nabla}$ ) and square phase (blue $\diamond$ ) show two peaks.

potential acting on the particles which is shown in Fig. 2. The half width of the interaction potential in the inset of Fig. 2 is $4.1 \mu \mathrm{m}$ which is somewhat larger than the actual half width of the optical line due to the finite size of the particles. ${ }^{25}$ As expected, ${ }^{20,25}$ the potential amplitude $V_{0}$ increases linearly with the laser intensity.

\subsection{Characterization of phases}

To distinguish between different structural phases obtained in our experiments we determined the particle-center coordinates by means of digital video microscopy ${ }^{26}$ and a particle-tracking algorithm in order to calculate the corresponding pair correlation functions $g(x, y)$. Fig. 3 shows a schematic illustration of the central region of $g(x, y)$ together with the trapping potential indicated by dashed vertical lines. From the distances $v$ and $u$ of the central particle to its nearest neighbours perpendicular and along the laser lines respectively, we define the order parameter $\xi=u / v$ which allows us to distinguish different structural phases according to Table 1.

\section{Results and discussions}

The experiments discussed in the following were performed with samples having a particle density $\rho \cong 0.0057 \mu \mathrm{m}^{-2}$. Each experiment lasted for 16 minutes and was recorded with a rate of $1 \mathrm{fps}$.

In the absence of the external potential, at this density the system forms a spontaneous crystal with random orientation 

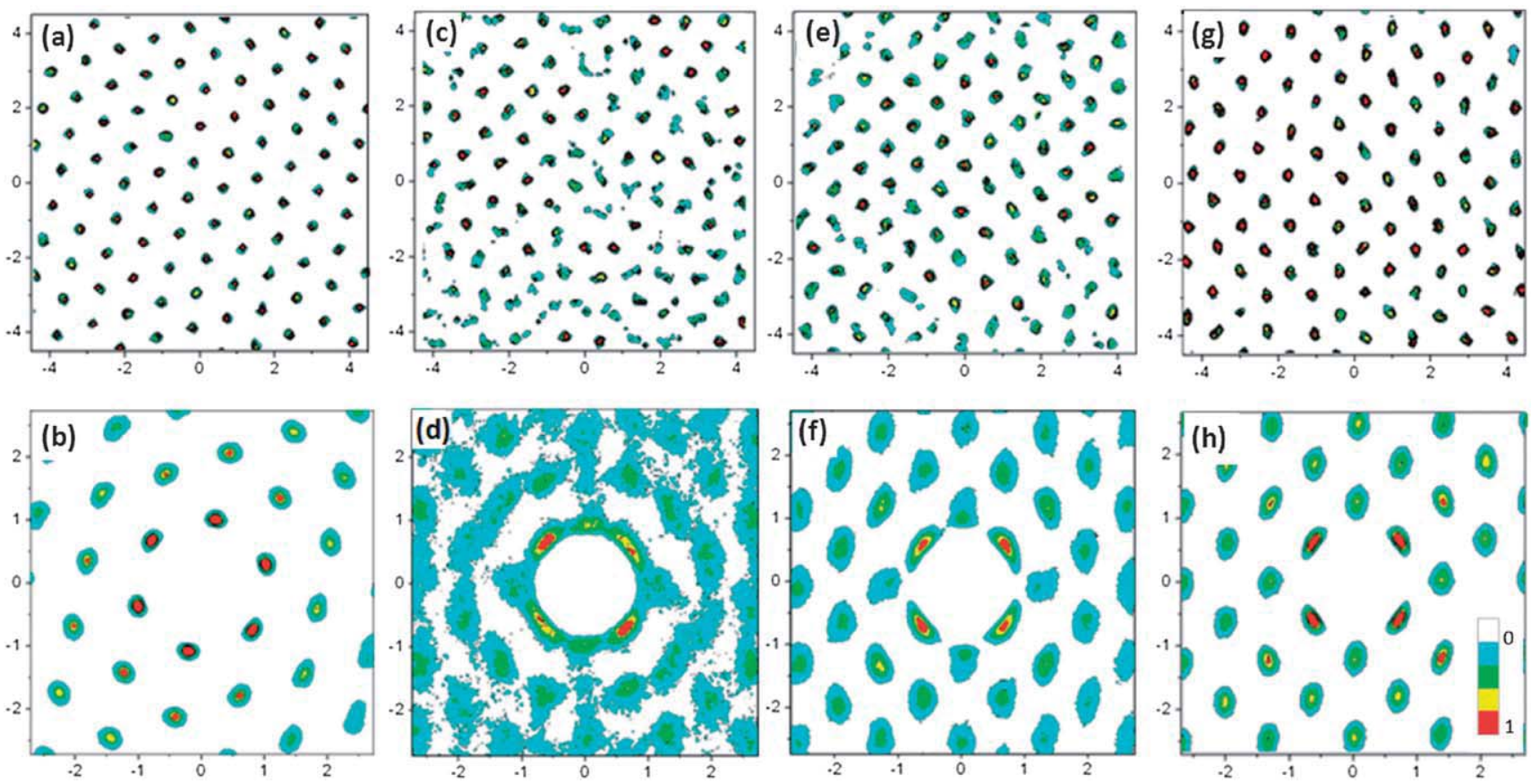

Fig. $6 \rho(x, y)$ and $g(x, y)$ are shown at the top and bottom respectively at a fixed periodicity $\boldsymbol{d}=9.58 \mu \mathrm{m}$ and different potential strengths for a randomly oriented triangular crystal. ( $\mathrm{a}$ and $\mathrm{b}$ ) at $V_{0}=0$, (c and d) a disordered state at $V_{0}=2.4 k_{\mathrm{B}} T$, a square phase shown in (e and f) at $V_{0}=3.6 k_{\mathrm{B}} T$ and $\left(\mathrm{g}\right.$ and $\mathrm{h}$ ) at $V_{0}=7.2 k_{\mathrm{B}} T$. All length scales are normalized by the lattice constant $\mathbf{a}$ of the triangular crystal.

and a mean lattice constant of about $13.5 \mu \mathrm{m}$. Fig. 4 shows the corresponding snap shots as well as the particle densities and pair correlations when the periodicity of the laser pattern was set to values between 6 and $12 \mu \mathrm{m}$. Each measurement was started from a spontaneous crystal $\left(V_{0}=0\right)$ and the intensity was slowly increased to $7.2 k_{\mathrm{B}} T$. Due to this protocol, the particles can initially exchange between potential lines and the obtained structures correspond to equilibrium phases. In Fig. 4(a)-(c) the periodicity is tuned to the triangular commensurate case $\boldsymbol{d} / \boldsymbol{a}=0.86$. At $V_{0}=1.5 k_{\mathrm{B}} T$ the crystal aligns accordingly and eventually forms a perfect triangular I structure at $7.2 k_{\mathrm{B}} T$. Fig. $4(\mathrm{~d})-(\mathrm{f})$ correspond to $\boldsymbol{d} / \boldsymbol{a}=0.77$. According to the above definition of phases, under such conditions a rhombic I phase with $\xi=0.83$ is obtained. Fig. $4(\mathrm{~g})$-(i) correspond to the square commensurate phase where $\boldsymbol{d} / \boldsymbol{a}=0.71$. Further decrease of the line spacing to $\boldsymbol{d} / \boldsymbol{a}=0.63$ leads to the rhombic phase II Fig. 4(j)-(l) and finally, for $\boldsymbol{d} / \boldsymbol{a}=0.5$ a rotated triangular crystal (triangular II phase) is obtained as in Fig. 4(m)-(o).The reason for the formation of the different phases is the counter play between optical trapping forces and the repulsive pair potential. While the optical forces align the particles along the potential lines at a given distance $\boldsymbol{d}$, the strong electrostatic particle repulsion leads to a registration between adjacent lines. It is important to realize, that due to the (a)

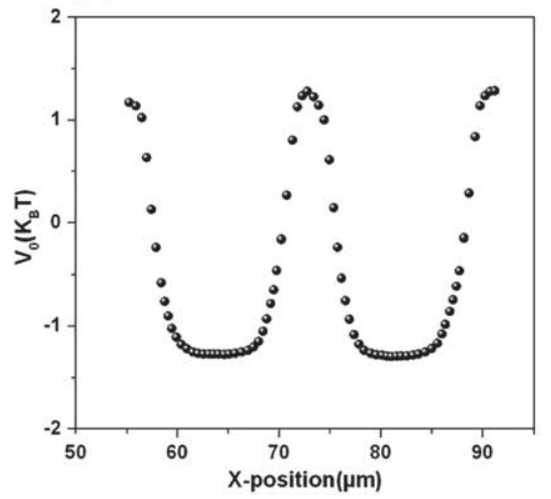

(b)

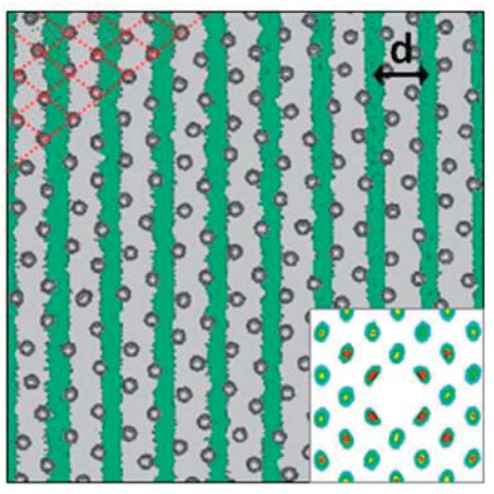

Fig. 7 (a) Part of a non-symmetric potential cross-section for a periodicity of $18 \mu \mathrm{m}$ and a width of the potential wells of $11.5 \mu \mathrm{m}$. (b) Snapshot of colloidal particles superimposed to the potential pattern with potential wells (grey) and laser lines (green). The potential depth was set to $8.3 k_{\mathrm{B}} T$. Red lines show the colloidal structure and the inset with the corresponding $g(x, y)$ gives $\xi=0.85$ indicating a rhombic I phase. 
constant total particle density in the sample cell, the particle line density will change upon variation of the line spacing $\boldsymbol{d}$. For example, during the transition from the triangular I to the square phase, i.e. upon decreasing the distance $\boldsymbol{d}$, the particle density along the lines will decrease. Note, that the length of the laser lines in $y$-direction is about $3 \mathrm{~mm}$ being much smaller than the extension of the sample cell which allows the exchange of particles in that direction.

The different phases can be also clearly distinguished by analysing their bond-length histogram. Bond-lengths were calculated based on a Delaunay triangulation ${ }^{27,28}$ that identifies the bonds between each particle and its nearest neighbour. Fig. 5 shows such normalized histograms for the different phases taken at $V_{0}=7.2 k_{\mathrm{B}} T$. Both, the triangular I and the triangular II phases show a single peak corresponding to sixfold coordinated particles at distance $\boldsymbol{a}$. In contrast, the rhombic I and II phases show a decrease in the amplitude of the first peak and a rise of a second one at a larger distance. The first peak corresponds to 4 -fold coordinated particles at distance $\boldsymbol{a}$ and the second peak corresponds to 2 -fold coordinated particle at distances between $\boldsymbol{a}$ and less than $\boldsymbol{a} \sqrt{3}$. For the square phase, also two peaks are observed, one corresponding to the four nearest neighbours at distance $\boldsymbol{a}$ and a second peak at distance $\boldsymbol{a} \sqrt{2}$. Due to the quasiperiodic order in two-dimensional systems, in general the second peak of the bond-length histograms is slightly broader than that of the first peak.

In Fig. 6 we have investigated how the square phase gradually forms for a period $\boldsymbol{d}=9.58 \mu \mathrm{m}$ with increasing the laser intensity. Starting from a randomly oriented spontaneous triangular crystal at zero light intensity as shown in Fig. 6a and $\mathrm{b}$, we observe at $V_{0}=2.4 k_{\mathrm{B}} T$ an intermediate phase as shown in Fig. $6 \mathrm{c}$ and d. By looking particularly at the pair correlation function, which indicates the probability to find a particle at a certain position, a ring is observed around the center. This is a signature of an isotropic distribution of the particles and therefore a liquid state. At the same time four peaks develop on this ring indicating a higher probability to find particles at these positions, giving some local order in the system. Therefore we describe this phase as a disordered state. This is caused by the light potential which is, in this case, not strong enough to force registration of particles and the square phase is not able to form yet. Increasing the potential strength localizes the particles in the potential wells and enhances their positional order which finally leads to a perfect square phase (Fig. 6(e)-(h)).

As already pointed out, the creation of potential landscapes by a scanned laser line offers several advantages compared to interference patterns. This includes the creation of non-periodic particle-light potentials as mentioned in Section 2.2, and also periodic but non-symmetric patterns. The latter is achieved by replacing the even step-like voltage protocol shown in Fig. 1d (corresponding to a single distance of the scanned laser lines) by an uneven step function applied to the voltage controlled mirror (corresponding to two distances including a small one in the order of $1 \mu \mathrm{m})$. This is exemplarily shown in Fig. 7a, where we create a non-symmetric particle-light interaction potential with periodicity of $18 \mu \mathrm{m}$ and a width of the potential wells of $11.5 \mu \mathrm{m}$. Applying this potential with a particle-light interaction strength of $6 k_{\mathrm{B}} T$ or above to a colloidal crystal of lattice constant $\boldsymbol{a}=12.5 \mu \mathrm{m}$ results in the formation of a rhombic I phase. In contrast to Fig. $4 \mathrm{~d}$ and e, however, here two rows of particles are trapped within a single optical potential line where they are arranged in a zig-zag pattern due to their electrostatic repulsion. Similar to above, a change in the periodicity of the light potential will result in a square phase.

\section{Conclusion}

We have reported on the creation of colloidal crystalline phases in one-dimensional periodic potential landscapes. In order to realize a wide range of line spacings relative to the lattice constant, we use a suspension of silica particles and bromobenzene as a solvent. Because the Debye screening length of the system is about $4.6 \mu \mathrm{m}$ it results in the formation of crystals with lattice constants up to $20 \mu \mathrm{m}$. In the presence of the laser pattern, the crystals are stabilized by the competition of optical and electrostatic forces and thus strongly depend on both the periodicity and the laser intensity of the light field. By using scanning optical tweezers, such potential landscapes can-in contrast to interference patterns-be controlled in situ without realignment of optical components.

\section{Acknowledgements}

We acknowledge the help of M. Thieman during sample preparation. This work is supported by the Marie Curie Initial Training Network Comploids, funded by the European Union Seventh Framework Program.

\section{References}

1 P. Pieranski, Colloidal Crystals, Contemp. Phys., 1983, 24(1), 25-73.

2 J. Kosterlitz and D. Thouless, J. Phys. C: Solid State Phys., 1973, 6, 1181.

3 A. van Blaaderen, R. Ruel and P. Wiltzius, Nature, 1997, 385, 321-324.

4 P. P. Lele and E. M. Furst, Langmuir, 2009, 25(16), 8875-8878.

5 A. Yethiraj and A. van Blaaderen, Nature, 2003, 421, 513-516.

6 M. Brunner, C. Bechinger, W. Strepp, V. Lobaskin and H. H. v. Grünberg, Europhys. Lett., 2002, 58, 926-965.

7 K. Mangold, P. Leiderer and C. Bechinger, Phys. Rev. Lett., 2003, 90(15), 158302.

8 M. Brunner and C. Bechinger, Phys. Rev. Lett., 2002, 88(24), 248302.

9 J. Mikhael, J. Roth, L. Helden and C. Bechinger, Nature, 2008, 454, 501-504.

10 A. Chowdhury, B. J. Ackerson and N. Clark, Phys. Rev. Lett., 1985, 55(8), 833-836.

11 J. Chakrabarti, H. R. Krishnamurthy, A. K. Sood and S. Sengupta, Phys. Rev. Lett., 1995, 75(11), 2236-2239.

12 Q.-H. Wei, C. Bechinger, D. Rudhardt and P. Leiderer, Phys. Rev. Lett., 1998, 81(12), 2606-2609.

13 R. K. Iler, The Chemistry of Silica, John Wiley \& Sons, NewYork, 1979. 
14 P. A. Thiessen and O. Koerner, Z. Anorg. Allg. Chem., 1930, 189, 168-173.

15 G. Zhang and I. Hua, Chemosphere, 2001, 46(2002), 59-66.

16 P. V. D. Hoeven, Electrostatic Stabilization of Suspensions in Non-Aqeous Media, 1991.

17 R. Evans, Adv. Phys., 1979, 28, 143-200.

18 K. Mecke, M. Oettel and R. Roth, J. Chem. Phys., 2012, 136, 081101.

19 J.-P. Hansen and I. R. McDonald, Theory of simple liquids, Academic press, London, 1986.

20 A. Ashkin, Phys. Rev. Lett., 1970, 24, 156-159.

21 A. Ashkin, Science, 1980, 210, 1081-1088.
22 D. G. Grier, Curr. Opin. Colloid Interface Sci., 1997, 2, 264270.

23 T. Tlusty, A. Meller and R. Bar-Ziv, Phys. Rev. Lett., 1998, 81(8), 1738-1741.

24 R. Lück, Int. J. Mod. Phys. B, 1993, 07, 1437-1453.

25 K. Loudiyi and B. J. Ackerson, Physica A, 1992, 184, 1-25.

26 J. C. Crocker and D. G. Grier, J. Colloid Interface Sci., 1996, 179, 298-310.

27 F. P. Preparata and M. I. Shamos, Computational Geometry, Springer, New York, 1985.

28 J. Mikhael, G. Gera, T. Bohlein and C. Bechinger, Soft Matter, 2011, 7, 1352-1357. 